\title{
A comparative study of embedded and anesthetized zebrafish in vivo on myocardiac calcium oscillation and heart muscle contraction
}

\author{
Brian S. Muntean ${ }^{1 \dagger}$, Christine M. Horvat ${ }^{1 \dagger}$, James H. Behler ${ }^{1}$, Wissam A. AbouAlaiwi' , Andromeda M. Nauli ${ }^{2}$, \\ Frederick E. Williams ${ }^{1}$ and Surya M. Nauli ${ }^{1}$ *
}

Department of Pharmacology, Colleges of Pharmacy and Medicine, The University of Toledo, Toledo, OH, USA

2 Department of Health Sciences, College of Public Health, East Tennessee State University, Johnson City, TN, USA

\section{Edited by:}

Ding-Feng Su, Second Military Medical

University, China

\section{Reviewed by:}

Huang-Tian Yang, Shanghai Jiao Tong

University, China

Lei-Ming Ren, HeBei University, China

${ }^{*}$ Correspondence:

Surya M. Nauli, Department of

Pharmacology, The University of

Toledo, MS 1015, Health Science

Campus, HEB 274, 3000 Arlington

Avenue, Toledo, $\mathrm{OH} 43614$, USA.

e-mail: surya.nauli@utoledo.edu

${ }^{\dagger}$ Brian S. Muntean and Christine M. Horvat have contributed equally on the project.
The zebrafish (Danio rerio) has been used as a model for studying vertebrate development in the cardiovascular system. In order to monitor heart contraction and cytosolic calcium oscillations, fish were either embedded in methylcellulose or anesthetized with tricaine. Using high-resolution differential interference contrast and calcium imaging microscopy, we here show that dopamine and verapamil alter calcium signaling and muscle contraction in anesthetized zebrafish, but not in embedded zebrafish. In anesthetized fish, dopamine increases the amplitude of cytosolic calcium oscillation with a subsequent increase in heart contraction, whereas verapamil decreases the frequency of calcium oscillation and heart rate. Interestingly, verapamil also increases myocardial contraction. Our data further indicate that verapamil can increase myocardial calcium sensitivity in anesthetized fish. Taken together, our data reinforce in vivo cardiac responses to dopamine and verapamil. Furthermore, effects of dopamine and verapamil on myocardial calcium and contraction are greater in anesthetized than embedded fish. We suggest that while the zebrafish is an excellent model for a cardiovascular imaging study, the cardio-pharmacological profiles are very different between anesthetized and embedded fish.

\section{INTRODUCTION}

The zebrafish (Danio rerio) has become an important model organism for studying various diseases, genetic variations, protein functions, behavioral selectivity, environmental toxicology, and many other physiological responses for high-throughput chemical screening (Carvan et al., 2004; Burns et al., 2005; Wessely and Obara, 2008; Ingham, 2009). Without doubt, the decision to sequence the entire genome of the zebrafish has helped in consolidating zebrafish as an experimental model (Vogel, 2000). In particular, zebrafish has been used for various genetic and pharmacological studies.

Although the zebrafish was originally used as a model for the study of vertebrate development, it has recently been used for other studies, largely due to its transparency. In many other animal models, in vivo physiological imaging of internal organs has remained relatively inaccessible. More specifically, calcium imaging using probes has been used in zebrafish in vivo (Huisken et al., 2004; Ebert et al., 2005; Milan et al., 2006; Arnaout et al., 2007; Chi et al., 2008) and confirmed in the isolated heart (Sehnert et al., 2002; Sedmera et al., 2003; Langenbacher et al., 2005). The patterns of free calcium have also been measured in zebrafish embryos (Creton et al., 1998).

Many laboratories have demonstrated substantial similarities in the pharmacological responses of the zebrafish heart to a broad range of pharmacological agents. More importantly, the therapeutic and toxic responses for both cardiac and non-cardiac drugs can be performed by simply immersing the fish in the intended drug (Langheinrich, 2003; Milan et al., 2003). However, accurate measurements of cardiovascular variables can only be achieved by immobilizing the fish during recordings.

In this study, we show that the two most used techniques to immobilize the fish can alter pharmacological responses differently, especially within the cardiovascular system. The effects of dopamine and verapamil on fish immobilized with methylcellulose (embedded) or tricaine (anesthetized) were analyzed. Dopamine and verapamil were selected, because their respective (+)- and (-)-inotropic actions on the heart have been well documented. In the present study, we simultaneously measured intracellular calcium in the heart and monitored the heart muscle in vivo in embedded and anesthetized zebrafish. We measure both myocardial calcium and heart contraction in terms of oscillations, frequencies, and amplitudes. In addition, we determine the effects of dopamine and verapamil on these variables.

\section{MATERIALS AND METHODS}

We used the wild type (WT) strain of zebrafish as our animal model, which was purchased from the Zebrafish International Resource Center (Eugene, OR, USA). The use of zebrafish was approved by The University of Toledo Animal Care and Use committee. All fish were studied at approximately 1 week old.

\section{ZEBRAFISH PREPARATION}

To enable cytosolic calcium measurement, the fish was incubated with $60 \mu \mathrm{g} / \mathrm{mL}$ fluo- $4 \mathrm{AM}$ or fura-2 AM (Invitrogen, Inc.) containing $2.5 \% \mathrm{DMSO}$ in egg water for $30 \mathrm{~min}$ at room temperature. 
The egg water was composed of $6 \% \mathrm{NaCl}$ in sterile distilled water (Carvan et al., 2004). Whereas fluo-4 was used to enable us to capture calcium oscillation at a greater rate $(\sim 1,560$ frames $/ \mathrm{min})$, fura-2 was utilized to obtain a better signal-to-noise fluorescence intensity. The fish was then rinsed twice with egg water and briefly monitored under a light microscope for heartbeat. At this stage, fish with any sign of abnormal heartbeat or agility in swimming were discarded. The healthy fish were then transferred in egg water containing $50 \mu \mathrm{g} / \mathrm{mL}$ tricaine (Sigma Aldrich, Inc.) for $30 \mathrm{~s}$.

For embedded preparation, sedated fish was mounted in $3 \%$ methylcellulose (Sigma Aldrich, Inc.). Note that it was necessary to briefly sedate the fish in this preparation for a better position and control of the fish on our microscope chamber. The fish was then placed on a custom-made plate with the depression of a number 1.0 coverglass in the center. The fish was orientated to its side to achieve a desired position for imaging. About $250 \mu \mathrm{L}$ methylcellulose was immediately dropped onto the fish to fill the plate depression, or until the fish was secure. Once the methylcellulose was solidified, usually in less than a minute at room temperature, the methylcellulose and fish were covered with egg water for the entire duration. Although the fish usually recovered in about $5 \mathrm{~min}$, we waited for at least $10 \mathrm{~min}$ to remove the residual tricaine by changing the egg water every $2 \mathrm{~min}$. Recovery of the fish was confirmed with a stable heartbeat of about 120 beats/min before any experiments were performed.

For tricaine preparation, we have titrated the incubation time (5 min) and concentration $(50 \mu \mathrm{g} / \mathrm{mL})$ of tricaine to be optimal to stabilize the fish for the duration of our experiments. Sedated fish were considered stable when limited physical movements were observed. The fish was then transferred to our customized plate with glass property optimal for our imaging studies. For the entire duration, the fish was covered with egg water.

\section{PHARMACOLOGICAL AGENTS}

Effects of dopamine (Acros Organics, Inc.) or verapamil (Sigma Aldrich, Inc.) on heart contractions were titrated with various concentrations of $0,1,4,10,40,100$, and $400 \mu \mathrm{g} / \mathrm{mL}$ in a total of 150 independent fish (Tables 1 and 2). For the rest of our studies, we chose a dosage of $100 \mu \mathrm{g} / \mathrm{mL}$ for dopamine or $4 \mu \mathrm{g} / \mathrm{mL}$ for verapamil, because these dosages were determined experimentally to be the suboptimal concentrations for their effects on the heart contractions in anesthetized fish. More important, these dosages provided an obvious difference in their physiological effects between methylcellulose-embedded and tricaine-treated fish. For all experiments, an equilibration time of at least $1 \mathrm{~min}$ with or without drug was achieved prior to image recording. In some experiments, propranolol (Sigma Aldrich, Inc.) were also used with a concentration of $0.001,0.01,0.1,1,3$, and $10 \mu \mathrm{M}$.

\section{IMAGE RECORDING}

The fish was imaged at the objective magnifications of $2 \times, 20 \times$, or $40 \times$ with the Nikon Eclipse TE2000-U microscope controlled with the Metaflor program as previously described (Nauli et al., 2008; AbouAlaiwi et al., 2009). We imaged the fish with all three magnifications for verification purposes. The $2 \times$ magnification would allow us to monitor the entire body of the fish. Whereas $20 \times$ would allow us to monitor heart and other organs concurrently (such
Table 1 | Effects of tricaine and verapamil on heart rate and calcium oscillation.

\begin{tabular}{|c|c|c|c|}
\hline $\begin{array}{l}\text { Tricaine } \\
\text { ( } \mu \mathrm{g} / \mathrm{mL})\end{array}$ & $\begin{array}{l}\text { Verapamil } \\
(\mu \mathrm{g} / \mathrm{mL})\end{array}$ & $\begin{array}{l}\text { Heart rate } \\
\text { (beats/min) }\end{array}$ & $\begin{array}{l}\mathrm{Ca}^{2+} \text { oscillation } \\
\text { (oscillations/min) }\end{array}$ \\
\hline \multirow[t]{7}{*}{0} & 0 & $120 \pm 4$ & $121 \pm 3$ \\
\hline & 1 & $123 \pm 3$ & $123 \pm 2$ \\
\hline & 4 & $116 \pm 4$ & $115 \pm 2$ \\
\hline & 10 & $103 \pm 6$ & $109 \pm 4$ \\
\hline & 40 & $52 \pm 10$ & $53 \pm 11$ \\
\hline & 100 & $0 \pm 0$ & $0 \pm 0$ \\
\hline & 400 & $0 \pm 0$ & $0 \pm 0$ \\
\hline \multirow[t]{7}{*}{10} & 0 & $122 \pm 5$ & $121 \pm 4$ \\
\hline & 1 & $122 \pm 5$ & $120 \pm 4$ \\
\hline & 4 & $102 \pm 10$ & $106 \pm 6$ \\
\hline & 10 & $86 \pm 12$ & $89 \pm 13$ \\
\hline & 40 & $18 \pm 7$ & $23 \pm 20$ \\
\hline & 100 & $0 \pm 0$ & $0 \pm 0$ \\
\hline & 400 & $0 \pm 0$ & $0 \pm 0$ \\
\hline \multirow[t]{7}{*}{20} & 0 & $120 \pm 5$ & $119 \pm 2$ \\
\hline & 1 & $116 \pm 6$ & $113 \pm 1$ \\
\hline & 4 & $102 \pm 11$ & $105 \pm 3$ \\
\hline & 10 & $65 \pm 7$ & $61 \pm 5$ \\
\hline & 40 & $12 \pm 5$ & $11 \pm 4$ \\
\hline & 100 & $0 \pm 0$ & $0 \pm 0$ \\
\hline & 400 & $0 \pm 0$ & $0 \pm 0$ \\
\hline \multirow[t]{7}{*}{30} & 0 & $118 \pm 2$ & $118 \pm 3$ \\
\hline & 1 & $100 \pm 5$ & $100 \pm 4$ \\
\hline & 4 & $90 \pm 11$ & $85 \pm 9$ \\
\hline & 10 & $64 \pm 6$ & $64 \pm 5$ \\
\hline & 40 & $20 \pm 12$ & $19 \pm 11$ \\
\hline & 100 & $0 \pm 0$ & $0 \pm 0$ \\
\hline & 400 & $0 \pm 0$ & $0 \pm 0$ \\
\hline \multirow[t]{7}{*}{40} & 0 & $81 \pm 8$ & $82 \pm 8$ \\
\hline & 1 & $70 \pm 8$ & $74 \pm 6$ \\
\hline & 4 & $50 \pm 10$ & $47 \pm 8$ \\
\hline & 10 & $26 \pm 26$ & $25 \pm 25$ \\
\hline & 40 & $11 \pm 11$ & $10 \pm 10$ \\
\hline & 100 & $0 \pm 0$ & $0 \pm 0$ \\
\hline & 400 & $0 \pm 0$ & $0 \pm 0$ \\
\hline \multirow[t]{7}{*}{50} & 0 & $68 \pm 6$ & $66 \pm 6$ \\
\hline & 1 & $66 \pm 9$ & $66 \pm 7$ \\
\hline & 4 & $42 \pm 9$ & $40 \pm 9$ \\
\hline & 10 & $25 \pm 23$ & $22 \pm 22$ \\
\hline & 40 & $0 \pm 0$ & $0 \pm 0$ \\
\hline & 100 & $0 \pm 0$ & $0 \pm 0$ \\
\hline & 400 & $0 \pm 0$ & $0 \pm 0$ \\
\hline
\end{tabular}

as the eye or fin used as an internal negative control), and a $40 \times$ magnification would provide a better resolution for calcium and muscle displacement analysis.

Muscle displacement, which is equivalent to muscle movement or heart contraction, was imaged with a high-resolution differential interference contrast (DIC) microscope. Cytosolic calcium images from fluo-4 were captured at excitation and emission of 490 and $516 \mathrm{~nm}$, respectively. Cytosolic calcium images from fura-2 were 
Table 2 | Effects of dopamine and propranolol on heart rate and calcium oscillation.

\begin{tabular}{lclc}
\hline $\begin{array}{l}\text { Dopamine } \\
(\boldsymbol{\mu g} / \mathbf{m L})\end{array}$ & $\begin{array}{l}\text { Propranolol } \\
(\boldsymbol{\mu} \mathbf{M})\end{array}$ & $\begin{array}{l}\text { Heart rate } \\
\text { (beats/min) }\end{array}$ & $\begin{array}{l}\mathbf{C a}^{2+} \text { oscillation } \\
\text { (oscillations/min) }\end{array}$ \\
\hline 0 & 0 & $120 \pm 6$ & $118 \pm 5$ \\
0 & 0.001 & $107 \pm 6$ & $105 \pm 6$ \\
100 & 0 & $132 \pm 6$ & $132 \pm 5$ \\
100 & 0.001 & $116 \pm 7$ & $115 \pm 7$ \\
100 & 0.01 & $103 \pm 6$ & $105 \pm 4$ \\
100 & 0.1 & $96 \pm 4$ & $98 \pm 3$ \\
100 & 1 & $40 \pm 9$ & $38 \pm 9$ \\
100 & 3 & $29 \pm 13$ & $25 \pm 10$ \\
100 & 10 & $0 \pm 0$ & $0 \pm 0$ \\
\hline
\end{tabular}

captured at an emission wavelength of $510 \mathrm{~nm}$ and excitation wavelengths of 340 and $380 \mathrm{~nm}$. In some cases, DIC and fluorescence images were captured simultaneously. When fura- 2 was used, fluorescence images were captured for at least $200 \mathrm{~s}$. Otherwise, images were capture at 26 frames/s for 3,000 frames.

\section{DATA ANALYSIS}

All measurements were done at the ventricular of the zebrafish's heart. To avoid any movement artifact, all our data were compared with the measurement of the eye and other organs, which serve as internal controls in each corresponding fish (Figure 2). To further avoid motion artifact, we also compared background fluorescence of hearts with and without calcium indicator. Furthermore, calcium analysis was done by first subtracting any background fluorescence. The frequency of heart oscillation was determined by measuring the peak-to-peak interval of the oscillations for both cytosolic calcium and heart contraction. The magnitude of heart oscillation was measured by analyzing the peak-to-valley amplitude of the oscillations in both cytosolic calcium and muscle contraction. Data comparisons were done using ANOVA test followed by Tukey post-test analysis. Data are shown as mean \pm standard error of mean with statistical power greater than 0.8 at $p<0.05$.

\section{RESULTS}

In the present study, we show that zebrafish is an excellent model to study biological reflex in response to pharmacological agents such as dopamine and verapamil (Figure 1). To enable us to monitor heart muscle in zebrafish, fish were either embedded in methylcellulose or anesthetized with tricaine. These techniques allow us to monitor heart muscle contraction, cytosolic calcium oscillation, and/or both. To examine the effects of inotropic agents on the fish heart, we used submaximal concentrations of both dopamine $(100 \mu \mathrm{g} / \mathrm{mL})$ and verapamil $(4 \mu \mathrm{g} / \mathrm{mL})$, which provide maximal effects in anesthetized fish without killing the fish.

Due to the transparent nature of the zebrafish, we were able to observe and record heart muscle displacement. Heart muscle movement was studied for about $200 \mathrm{~s}$. As expected, no changes in muscle displacement or calcium fluctuation (Figure 2) were seen in the eye or other organs of the fish. The oscillation movement of the heart was plotted against time in line graphs (Figure 3).
Treatment with dopamine or verapamil showed obvious changes in the patterns of heart contraction in anesthetized, but not in embedded, zebrafish.

Likewise, cytosolic calcium was concurrently studied for at least $200 \mathrm{~s}$. Line graphs were generated to observe the patterns of calcium oscillation in the zebrafish's heart (Figure 4). The average changes in cytosolic calcium were plotted against time. Consistent with the previous report (Huisken et al., 2004; Ebert et al., 2005; Milan et al., 2006; Arnaout et al., 2007; Chi et al., 2008), calcium oscillation was seen in the heart. At least $30 \mathrm{~s}$ after treatment with dopamine or verapamil, changes in amplitudes of calcium oscillation in the heart could be observed in anesthetized fish.

When the oscillation frequency of the heart contraction (Figure 5A) or cardiac cytosolic calcium (Figure 5B) was analyzed more closely, our data showed that neither dopamine $(100 \mu \mathrm{g} / \mathrm{mL})$ nor verapamil $(4 \mu \mathrm{g} / \mathrm{mL})$ had any effect in methylcellulose embedded fish. The oscillation frequencies of the heart contraction in control, dopamine, and verapamil groups are not statistically significant from one another. Likewise, there is no difference among control, dopamine, and verapamil groups on the frequency of the cytosolic calcium oscillation.

In anesthetized fish, dopamine caused a slight decrease in heart contraction frequency, and verapamil significantly repressed the oscillation frequency of the heart contraction (Figure 5C). The frequency of cardiac contraction was significantly repressed in the verapamil-treated group, as compared to the control group. The dopamine-treated group did not show a significant change in the frequency of myocardial contraction. Whereas effect of dopamine was minimal, verapamil significantly repressed calcium frequency, compared to the control group (Figure 5D). The verapamil-treated group showed a repressed calcium frequency compared to the control group. The dopamine-treated group did not show a significant decrease in the frequency of myocardial contraction.

When the oscillation amplitude of the heart contraction (Figure 5E) or cardiac cytosolic calcium (Figure 5F) was analyzed, our data showed that neither dopamine nor verapamil had any effect in methylcellulose embedded fish. The oscillation amplitudes of the heart contraction in control, dopamine, and verapamil groups are not statistically significant from one another. Likewise, there is no difference among control, dopamine, and verapamil groups on the amplitude of the cytosolic calcium oscillation.

In anesthetized fish, it is interesting to note that both dopamine and verapamil induced a significant increase in magnitude of heart muscle displacement (Figure 5G). The magnitude of cardiac contraction was significantly greater in dopamine ( $2.00 \pm 0.40$ unit) and verapamil-treated groups as compared to the control group. Whereas dopamine increased magnitude of calcium oscillation, verapamil significantly repressed it (Figure 5H). Compared to the control group, the amplitude of calcium oscillation was increased in the dopamine-treated group and decreased in the verapamiltreated group.

While the frequency relationships between cytosolic calcium and heart contraction were consistent, the amplitude correlations between heart contraction and cytosolic calcium were unexpected in anesthetized fish (Figure 5). This is especially the case in the verapamil-treated group. We have previously shown that the changes 


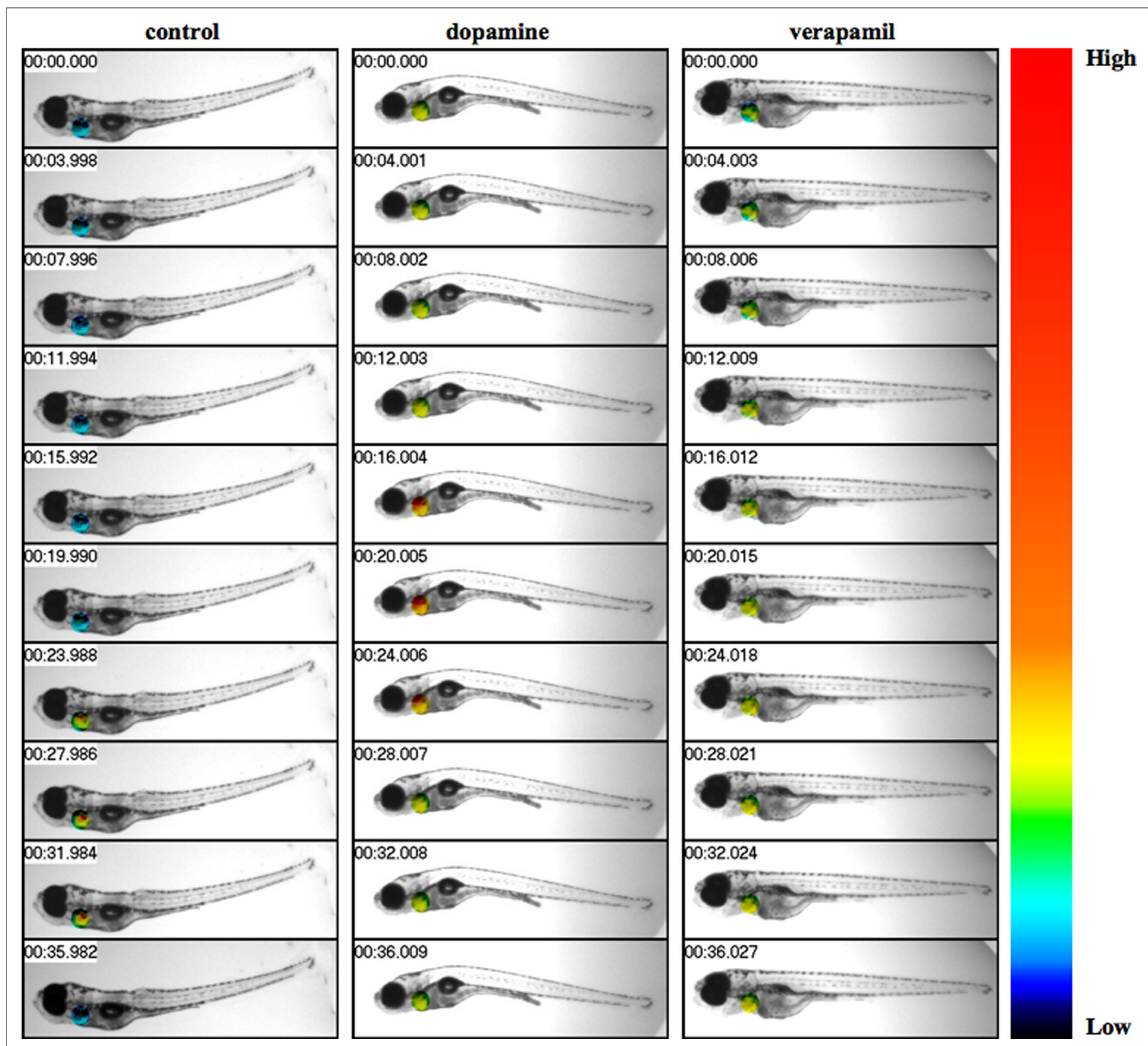

FIGURE 1 | Live-imaging effects of dopamine and verapamil are studied in zebrafish heart. Fish was treated with vehicle (control), dopamine, or verapamil. The intracellular calcium and muscle oscillation of the heart were monitored and recorded. Fish heart was pseudocolored to show the changes in intracellular calcium, as indicated in color bar where low to high intracellular calcium levels were denoted in black and red colors, respectively. Images were captured at a rate of either 26 frames/s. The changes in cytosolic calcium are shown for about $36 \mathrm{~s}$ at $4 \mathrm{~s}$ intervals, as indicated at the top left corner. in the contractile force for a given cytosolic calcium concentration can be altered during cerebral development (Nauli et al., 2001a,b). The relationship between contractile force and cytosolic calcium is known as calcium sensitivity. Surprisingly, our data show that verapamil significantly increased calcium sensitivity in the anesthetized zebrafish heart (Figure 6). In embedded fish, calcium sensitivity was not changed with treatment of either dopamine or verapamil, compared to the control group. In anesthetized fish, the calcium sensitivity in the verapamil-treated group is significantly greater than in the control group, whereas no difference is observed in the dopamine-treated group.

\section{DISCUSSION}

In the present study, we show that tricaine-anesthetized zebrafish becomes more sensitive in response to cardiovascular pharmacological agents. As we understand it, we are the first to examine the effects of pharmacological agents on both cardiac cytosolic calcium and myocardial contraction simultaneously in vivo in nontransgenic 1-week-old zebrafish (Figure 1). We also demonstrate that pharmacological agents, such as verapamil, have the potential to change myocardial calcium sensitivity in anesthetized fish. To our knowledge, we provide the first in vivo evidence that verapamil may have a more complicated physiological reflex than simply blocking 


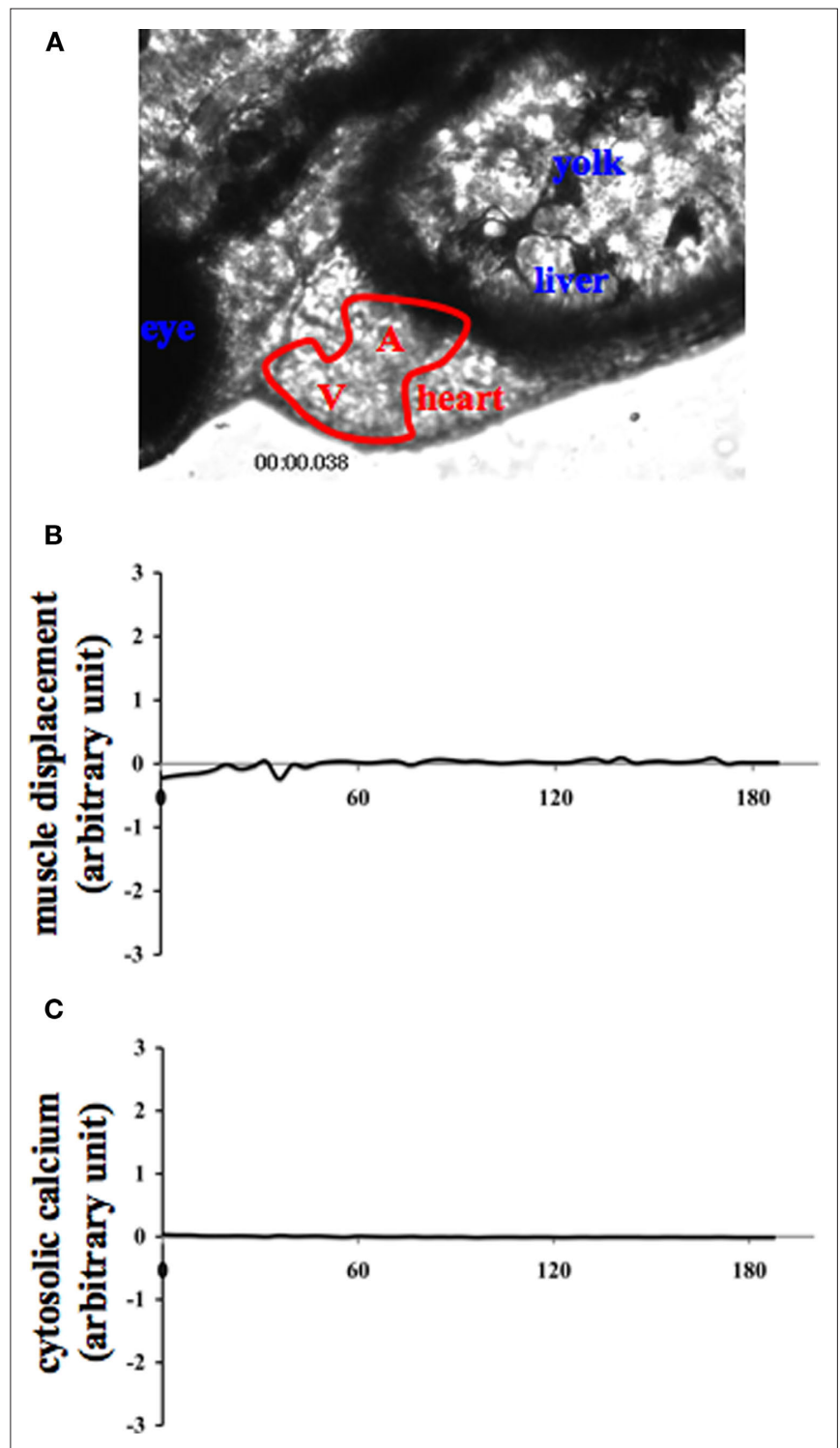

FIGURE 2 | Internal muscle movement and cytosolic calcium are studied in various organs of zebrafish. Internal organs of zebrafish were indicated by the corresponding text (A). A and $\mathrm{V}$ denote the atrium and ventricle of the heart, respectively. To verify the specificity of muscle contraction and calcium oscillation in the heart and to avoid any movement artifact, changes in muscle movement (B) and cytosolic calcium (C) of the corresponding fish were also measured in the eye. The eye measurement can therefore represent an internal negative control for cytosolic calcium recording.

calcium channels. We have therefore offered a new way to further these studies with concurrent measurements of heart cytosolic calcium and its role in myocardial contractility not only in developing fish but also in adult fish.

To enable us to monitor the heart of living fish, the fish was either embedded in methylcellulose or anesthetized with tricaine. In particular, tricaine is a frequently used as a general anesthetic for fish (Machin, 1999; Kolanczyk et al., 2003; Craig et al., 2006). This anesthetic agent is known to depress neuronal activity in the central and peripheral nervous systems (Machin, 1999; Kolanczyk et al., 2003; Craig et al., 2006). In addition, tricaine is highly lipid-soluble, and therefore is rapidly absorbed and eliminated by diffusion across the membranes of the gills (Kolanczyk et al., 2003). Tricaine is classified as a muscle relaxant that functions by blocking action potentials, therefore causing a lack of signals being exchanged between the brain and surrounding areas (Kolanczyk et al., 2003). With the lack of action potentials, no sensory input or muscle contractions are present. For this reason, tricaine is primarily used to temporarily immobilize fish, amphibians, and other aquatic cold-blooded animals to enable handling of these species during research.

At the correct concentration, tricaine can also be used for euthanasia. For our purposes, we used tricaine at a concentration of $50 \mu \mathrm{g} / \mathrm{mL}$ to enable monitoring of heart rate and contractility. This concentration of tricaine was optimal for the intended purpose. At this optimal concentration of tricaine, we observed a significant decrease in the heart rate of the zebrafish. Our experiences with tricaine show that it has a rapid anesthetic effect as well as a rapid recovery rate.

It has been shown that anesthesia can produce depressing effects on the cardiovascular system in both animal models and humans. For example, a study found that propofol moderately compromised cardiac contractility and increased afterload on the right ventricle, without significant effect on coronary perfusion pressure (Machala and Szebla, 2008). Although most cardiovascular compromise was clinically insignificant in the majority of general anesthetic, including propofol, certain combinations of anesthetic agents with other cardiovascular agents can cause additional effects or adverse effects. Our data, in particular, demonstrate that general anesthetic and combination of cardiovascular agents would increase sensitivity toward cardiovascular response in fish. The mechanism by which this occurs may involve myofilament calcium sensitivity of heart muscle.

Dopamine is known to be a positive inotropic agent that acts on the dopaminergic receptors (Ricci et al., 1998). In the myocytes, the dopaminergic receptor (D4) activation, which is a $\mathrm{G}_{\mathrm{i}}$-coupled receptor, will promote an increase in cytosolic calcium through a complex pathway involving the phospholipase $\mathrm{C}$, $\mathrm{IP}_{3} / \mathrm{IP}_{3}$ calcium channel and the plasmalemmal calcium channel through the $\mathrm{G}_{\beta \gamma}$ subunit (Ricci et al., 1998). The increase in intracellular calcium, which is a second messenger for the myosin-actin cross-bridge formation, will ultimately increase the contractile force. The inotropic characteristic of dopamine has been used in patients with advanced heart failure (Carey and Jacob, 1989). Consistent with this notion, dopamine increases myocardial muscle contraction in anesthetized fish (Figures 3 and 5). The enhanced heart muscle contraction is likely caused by dopamineinduced cytosolic calcium increases (Figures 3-5). It is important to note that the rates and frequency of calcium oscillation are not significantly altered by dopamine. Consequently, dopamine does not significantly alter the frequency of heart contraction. Our data support the view that dopamine increases myocardial contractility and cardiac output without changing heart rate (Contreras et al., 2002).

Verapamil belongs to the phenylalkylamine class of L-type calcium channel blockers. Verapamil has been used to treat cardiac arrhythmias, hypertension, and angina (Prisant, 2001), probably because the phenylalkylamine class has more selectivity in the heart than in peripheral vasculatures. Due to its site selectivity, we selected 

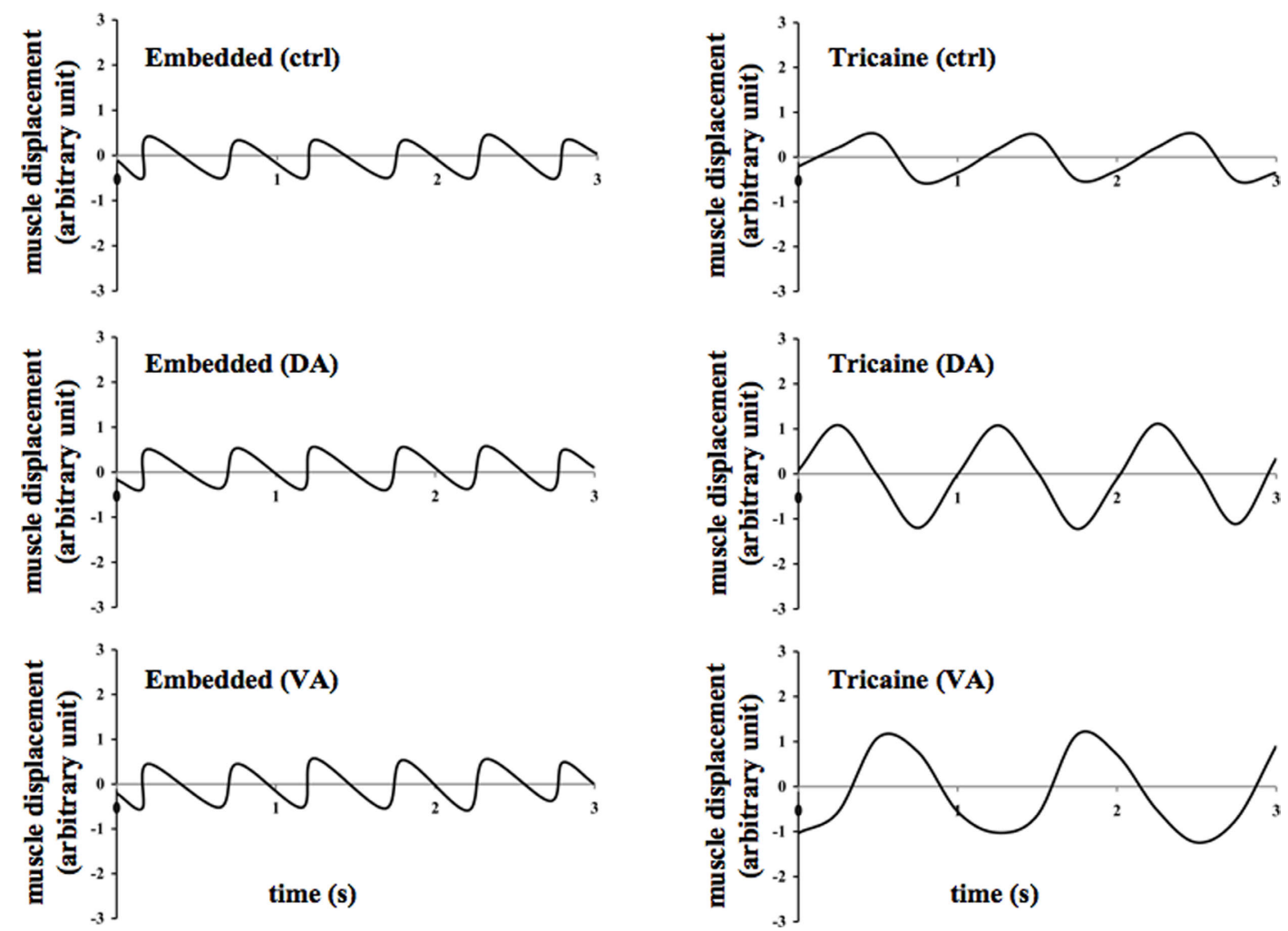

FIGURE 3 | Effects of dopamine and verapamil on heart muscle contraction were examined in the zebrafish. Representatives of heart muscle displacement are shown in the embedded and anesthetized fish treated with vehicle (ctrl), dopamine (DA), or verapamil (VA).
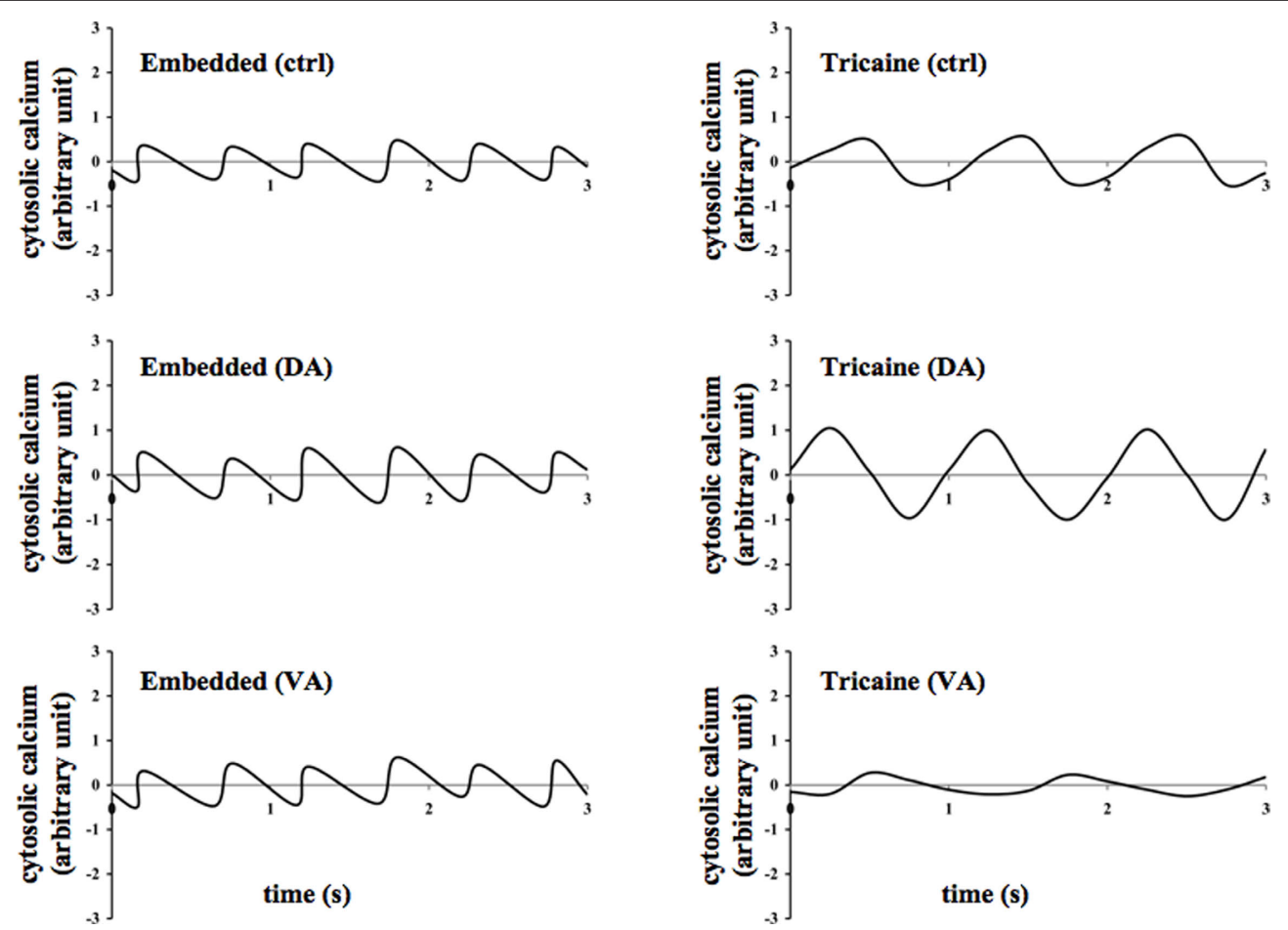

FIGURE 4 | Effects of dopamine and verapamil on cytosolic calcium oscillation were examined in the zebrafish. Representatives of calcium oscillation in the heart are shown in the embedded and anesthetized fish treated with vehicle (ctrl), dopamine (DA), or verapamil (VA). 
A

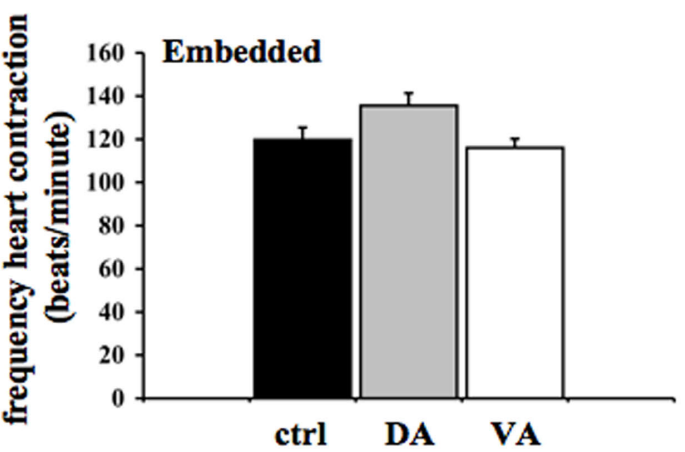

C

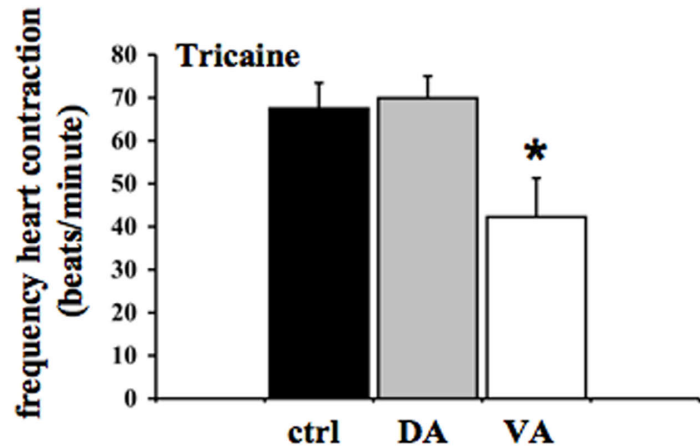

E
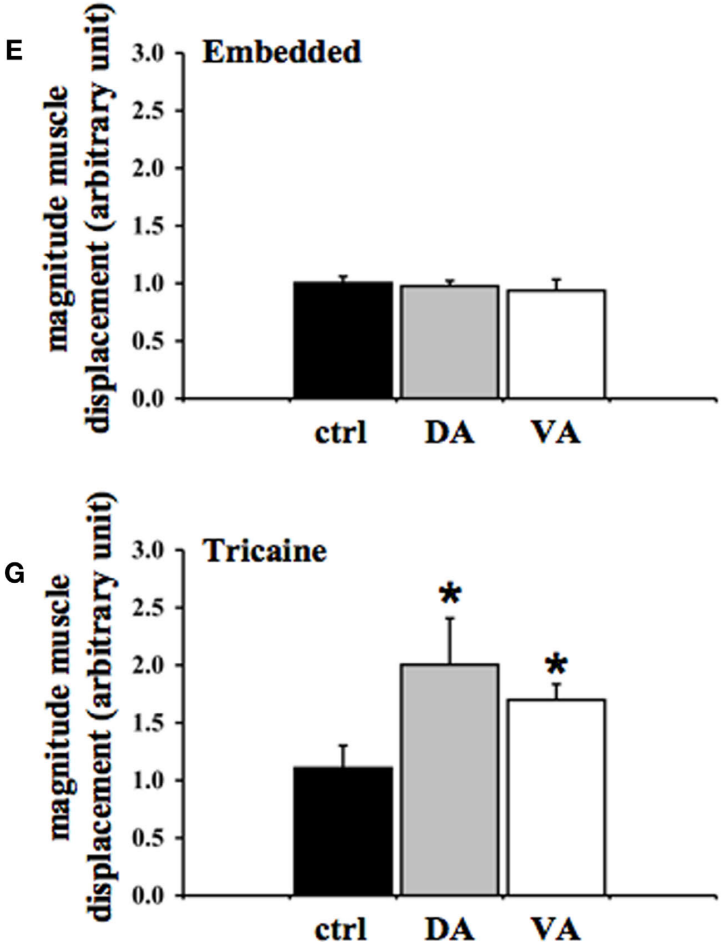

FIGURE 5 | Effects of dopamine and verapamil on frequency and amplitude of cytosolic calcium and muscle contraction are quantified in zebrafish heart. The frequency of the heart oscillation was measured by analyzing the peak-to-peak interval of the oscillations in embedded $(\mathbf{A}, \mathbf{B})$ and anesthetized (C,D) fish. The frequencies of muscle contraction $(\mathbf{A}, \mathbf{C})$ and cytosolic calcium $(\mathbf{B}, \mathbf{D})$ were averaged in fish treated with the vehicle (ctrl), dopamine (DA), or verapamil (VA). Verapamil significantly decreases the frequency of oscillations of both cytosolic calcium and muscle contraction in anesthetized fish. The
B

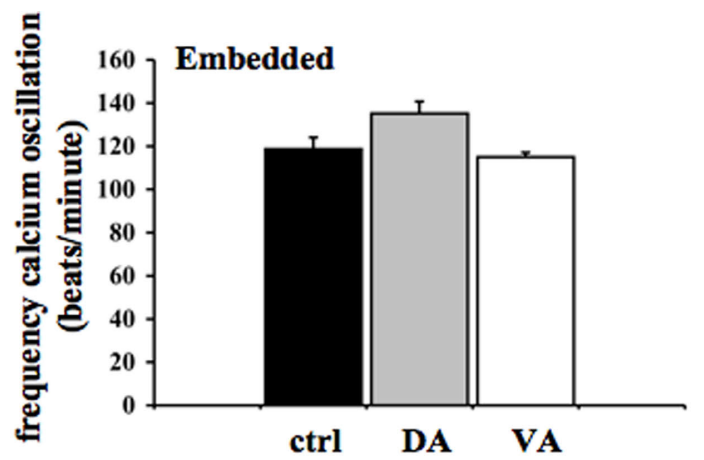

D
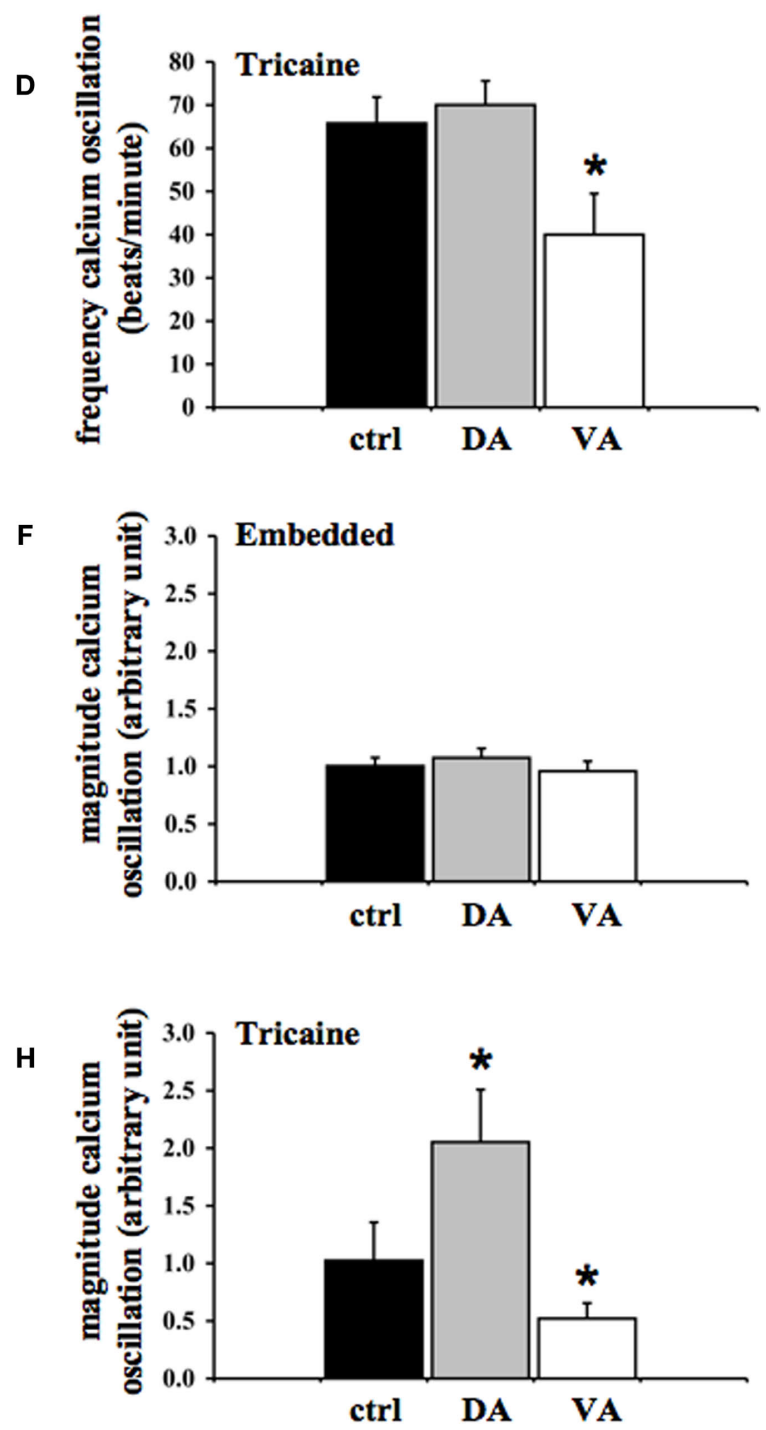

magnitude of the heart oscillation was measured by analyzing the peak-to-valley amplitude of the oscillations in embedded $\mathbf{( E , F )}$ and anesthetized $\mathbf{( G , H )}$ fish. The magnitudes of muscle contraction $\mathbf{( E , G ) ~ a n d ~ c y t o s o l i c ~ c a l c i u m ~} \mathbf{( F , H ) ~ w e r e ~}$ averaged accordingly. Both dopamine and verapamil significantly increase the magnitude of heart muscle contraction in the anesthetized zebrafish heart. While dopamine increases the amplitude of calcium, verapamil decreases the calcium amplitude in anesthetized fish. Asterisks denote a significant difference compared to control. $N=6$. 


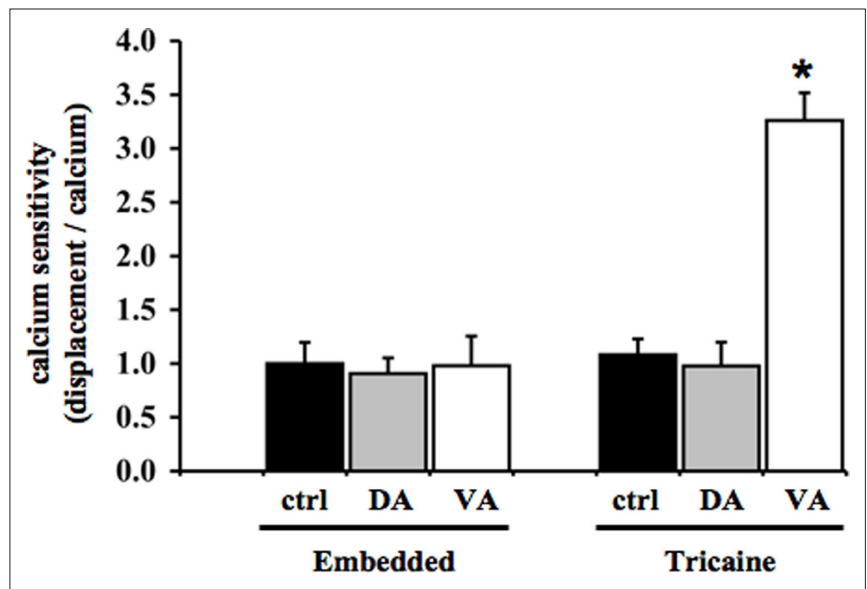

FIGURE 6 | Verapamil causes a physiological reflex that induces calcium sensitivity in anesthetized zebrafish heart. Calcium sensitivity is defined as the contractile force produced by a given increase in cytosolic calcium. When the values of contractile forces were divided by the changes in cytosolic calcium, the myogenic calcium sensitivity increased significantly only in anesthetized fish treated with verapamil. This indicates that verapamil as a calcium channel blocker has a greater complexity in the myogenic heart contractility in response to anesthetic. Asterisk denotes a significant difference compared to control.

verapamil as a pharmacological candidate in our study. Our data show that verapamil decreases the frequency of calcium oscillation in the anesthetized zebrafish heart (Figures 4 and 5). Subsequently, verapamil's inhibitory effect on cytosolic calcium reduces myocardial heartbeat (Figure 5). It is expected that verapamil also decreases the amplitude of cytosolic calcium oscillation in the anesthetized zebrafish heart. However, it surprises us that verapamil significantly increases myocardial contraction. Reflex vasoconstriction has been proposed to be a compensatory mechanism for verapamil. Here we propose that verapamil can also induce reflex myocardial inotropic effects, at least in the zebrafish.

To help explain the molecular coupling of cytosolic calcium and myofilament contractility for verapamil, we revisit the molecular theory termed "calcium sensitivity." The term explains that the efficiency of calcium-induced contraction can be regulated at the molecular level (McDaniel et al., 1994; Murphy and Walker, 1998). In particular, we and others have shown that the efficiency of utilizing calcium for contraction can be observed during cardiovascular development (Nauli et al., 2001a,b), pregnancy (Xiao et al., 2006), physiological perturbation (Mulvany and Nyborg, 1980), and so on. More importantly, physiological perturbation by verapamil also increases calcium sensitivity (Figure 6). Interestingly, dopamine does not promote significant changes in calcium sensitivity. One possible explanation is that a physiological reflex in response to verapamil has been initiated in the heart by tricaine to increase the heart muscle contraction. This reflex is probably necessary to prevent cardiovascular collapse due to a decreased heartbeat. Undoubtedly, this interesting phenomenon warrants more detailed studies in the future.

The effect of verapamil was further confirmed in anesthetized fish by using different tricaine concentrations to examine changes in heart rate and calcium oscillation (Table 1).
As it is apparently shown, increasing tricaine concentrations will enhance sensitivity of the fish to verapamil. Furthermore, because dopamine may also act through stimulation of betaadrenergic receptors, propranolol was used to analyze this possibility (Table 2). Our data exclude the possibility that dopamine acts through adrenergic receptors, because propranolol still shows a dose response effect on the heart in the presence of dopamine.

We use the 1-week-old zebrafish in our study for several reasons. First, the zebrafish at this age still have a transparent characteristic that provides tremendous opportunity to study their internal organs. This characteristic provides us with the ability to measure heart contractility and cytosolic calcium. Second, the 1-week-old zebrafish has developed a well-defined cardiovascular system. Thus, the 1-week-old zebrafish shows a strong heartbeat and welldefined cardiac muscle. Third, the 1-week-old zebrafish has mature cardiovascular responses, including responses to dopamine and verapamil observed in our experiments. In addition, the mature cardiovascular characteristics provide us with an advantage to study cardiovascular reflexes seen in the mammalian system. We believe that verapamil, which lowers the cytosolic calcium oscillation in our experiments, also generates a reflex by increasing inotropic heart contraction.

Although myocardial calcium of the zebrafish has been previously studied, only the isolated heart was examined (Sehnert et al., 2002; Sedmera et al., 2003; Langenbacher et al., 2005). The in vivo heart was studied in very young fish at about $48 \mathrm{~h}$ postfertilization (Huisken et al., 2004; Ebert et al., 2005; Milan et al., 2006; Arnaout et al., 2007; Chi et al., 2008). More importantly, no measurement of heart muscle displacement was ever recorded. However, one disadvantage of using an adult fish is that the anesthetization would significantly slow the heartbeat (Barrionuevo and Burggren, 1999). Despite this disadvantage, we would argue that the heart becomes more sensitive in response to pharmacological agents, as evidenced by our study.

Overall, our study reinforces the roles of dopamine and verapamil in cardiac myocytes. We show that tricaine-anesthetized zebrafish become more sensitive in response to cardiovascular pharmacological agents, such dopamine and verapamil. Our data also suggest a possible verapamil-induced inotropic reflex through an increase in myocardial calcium sensitivity in anesthetized zebrafish.

\section{ACKNOWLEDGMENTS}

This work was funded by awards from the AHA (0825195F), NIH (DK080640) and the NIH Recovery Act Funds for Administrative Supplements Providing Summer Research Experiences for Students and Science Educators. The completion of this work by Christine M. Horvat and James H. Behler partially fulfilled the requirements for the undergraduate practicum in pharmacology and toxicology for a Bachelor of Science degree in pharmaceutical sciences. Authors would like to thank Maki Takahashi and Blair Mell for their technical support and Charisse Montgomery for her editing assistance. The abstract of this work was presented at the Experimental Biology 2009 in New Orleans, LA, USA. 


\section{REFERENCES}

AbouAlaiwi, W. A., Takahashi, M., Mell, B. R., Jones, T. J., Ratnam, S., Kolb, R. J., and Nauli, S. M. (2009). Ciliary polycystin-2 is a mechanosensitive calcium channel involved in nitric oxide signaling cascades. Circ. Res. 104, 860-869.

Arnaout, R., Ferrer, T., Huisken, J., Spitzer, K., Stainier, D. Y., Tristani-Firouzi, M., and Chi, N. C. (2007). Zebrafish model for human long QT syndrome. Proc. Natl. Acad. Sci. U.S.A. 104, 11316-11321.

Barrionuevo, W. R., and Burggren, W. W. (1999). O2 Consumption and heart rate in developing zebrafish (Danio rerio): influence of temperature and ambient O2. Am. J. Physiol. 276, R505-513.

Burns, C. G., Milan, D. J., Grande, E. J., Rottbauer, W., MacRae, C. A., and Fishman, M. C. (2005). Highthroughput assay for small molecules that modulate zebrafish embryonic heart rate. Nat. Chem. Biol. 1, 263-264.

Carey, R. A., and Jacob, L. (1989). The role of dopaminergic agents and the dopamine receptor in treatment for CHF. J. Clin. Pharmacol. 29, 207-211.

Carvan, M. J. III, Loucks, E., Weber, D. N., and Williams, F. E. (2004) Ethanol effects on the developing zebrafish: neurobehavior and skeletal morphogenesis. Neurotoxicol. Teratol. 26, 757-768.

Chi, N. C., Shaw, R. M., Jungblut, B., Huisken, J., Ferrer, T., Arnaout, R., Scott, I., Beis, D., Xiao, T., Baier, H., Jan, L.Y., Tristani-Firouzi, M., and Stainier, D. Y. (2008). Genetic and physiologic dissection of the vertebrate cardiac conduction system. PLoS Biol. 6, e109. doi: 10.1371/journal.pbio.0060109.

Contreras, F., Fouillioux, C., Bolivar, A., Simonovis, N., HernandezHernandez, R., Armas-Hernandez, M. J., and Velasco, M. (2002). Dopamine, hypertension and obesity. J. Hum. Hypertens. 16(Suppl. 1), S13-S17.

Craig, M. P., Gilday, S. D., and Hove, J. R. (2006). Dose-dependent effects of chemical immobilization on the heart rate of embryonic zebrafish. Lab. Anim. 35, 41-47.

Creton, R., Speksnijder, J. E., and Jaffe, L. F. (1998). Patterns of free calcium in zebrafish embryos. J. Cell. Sci. 111(Pt 12), 1613-1622.

Ebert, A. M., Hume, G. L., Warren, K. S., Cook, N. P., Burns, C. G., Mohideen, M.A., Siegal, G., Yelon, D., Fishman, M. C., and Garrity, D. M. (2005). Calcium extrusion is critical for cardiac morphogenesis and rhythm in embryonic zebrafish hearts. Proc. Natl. Acad. Sci. U.S.A. 102, 17705-17710.

Huisken, J., Swoger, J., Del Bene, F., Wittbrodt, J., and Stelzer, E. H. (2004). Optical sectioning deep inside live embryos by selective plane illumination microscopy. Science 305 1007-1009.

Ingham, P. W. (2009). The power of the zebrafish for disease analysis. Hum. Mol. Genet. 18, R107-R112.

Kolanczyk, R. C., Fitzsimmons, P. N., McKim, J. M. Sr., Erickson, R. J., and Schmieder, P. K. (2003). Effects of anesthesia (tricaine methanesulfonate, MS222) on liver biotransformation in rainbow trout (Oncorhynchus mykiss). Aquat. Toxicol. 64, 177-184.

Langenbacher, A. D., Dong, Y., Shu, X. Choi, J., Nicoll, D. A., Goldhaber, J. I., Philipson, K. D., and Chen, J. N. (2005). Mutation in sodium-calcium exchanger 1 (NCX1) causes cardiac fibrillation in zebrafish. Proc. Natl. Acad. Sci. U.S.A. 102, 17699-17704.

Langheinrich, U. (2003).Zebrafish: a new model on the pharmaceutical catwalk. Bioessays 25, 904-912.

Machala, W., and Szebla, R. (2008). Effects of propofol induction on haemodynamics. Anestezjol. Intens. Ter. 40, 223-226.

Machin, K. L. (1999). Amphibian pain and analgesia. J. Zoo Wildl. Med. 30 2-10.

McDaniel, N. L., Rembold, C. M., and Murphy, R. A. (1994). Cyclic nucleotide dependent relaxation in vascular smooth muscle. Can. J. Physiol. Pharmacol. 72, 1380-1385.

Milan, D. J., Giokas, A. C., Serluca, F. C., Peterson, R. T., and MacRae, C. A. (2006). Notchlb and neuregulin are required for specification of central cardiac conduction tissue. Development 133, 1125-1132.

Milan, D. J., Peterson, T. A., Ruskin, J. N., Peterson, R. T., and MacRae, C. A. (2003). Drugs that induce repolarization abnormalities cause bradycardia in zebrafish. Circulation 107 1355-1358.

Mulvany, M. J., and Nyborg, N. (1980) An increased calcium sensitivity of mesenteric resistance vessels in young and adult spontaneously hypertensive rats. Br. J. Pharmacol. 71, 585-596.

Murphy, R. A., and Walker, J. S. (1998) Inhibitory mechanisms for crossbridge cycling: the nitric oxide-cGMP signal transduction pathway in smooth muscle relaxation. Acta Physiol. Scand $164,373-380$.

Nauli, S. M., Kawanabe, Y., Kaminski, J. J., Pearce, W. J., Ingber, D. E., and Zhou, J. (2008). Endothelial cilia are fluid shear sensors that regulate calcium signaling and nitric oxide production through polycystin-1. Circulation 117 1161-1171.

Nauli, S. M., Williams, J. M., Akopov, S. E. Zhang, L., and Pearce, W. J. (2001a). Developmental changes in ryanodine- and $\mathrm{IP}(3)$-sensitive $\mathrm{Ca}(2+)$ pools in ovine basilar artery. Am. J. Physiol. Cell Physiol. 281, C1785-C1796.

Nauli, S. M., Zhang, L., and Pearce, W. J. (2001b). Maturation depresses cGMP-mediated decreases in $[\mathrm{Ca} 2+]$ $\mathrm{i}$ and $\mathrm{Ca} 2+$ sensitivity in ovine cranial arteries. Am. J. Physiol. 280, H1019-H1028.

Prisant, L. M. (2001). Verapamil revisited: a transition in novel drug delivery systems and outcomes. Heart Dis. 3 , 55-62.

Ricci,A., Bronzetti, E., Fedele, F., Ferrante, F.,Zaccheo, D., and Amenta, F. (1998). Pharmacological characterization and autoradiographic localization of a putative dopamine D4 receptor in the heart. J. Auton. Pharmacol. 18, 115-121.

Sedmera, D., Reckova, M., deAlmeida A., Sedmerova, M., Biermann, M. Volejnik, J., Sarre, A., Raddatz, E., McCarthy, R. A., Gourdie, R. G., and Thompson, R. P. (2003). Functional and morphological evidence for a ventricular conduction system in zebrafish and Xenopus hearts. Am. J. Physiol. 284, H1152-H1160.

Sehnert, A. J., Huq, A., Weinstein, B. M., Walker, C., Fishman, M., and Stainier, D. Y. (2002). Cardiac troponin T is essential in sarcomere assembly and cardiac contractility. Nat. Genet. 31, 106-110.

Vogel, G. (2000). Genomics. Sanger will sequence zebrafish genome. Science 290, 1671.

Wessely, O., and Obara, T. (2008). Fish and frogs: models for vertebrate cilia signaling. Front. Biosci. 13, 1866-1880.

Xiao, D., Huang, X., Longo, L. D., Pearce, W. J., and Zhang, L. (2006). Regulation of baseline $\mathrm{Ca} 2+$ sensitivity in permeabilized uterine arteries: effect of pregnancy. Am. J. Physiol. 291, H413-H420.

Conflict of Interest Statement: The authors declare that the research was conducted in the absence of any commercial or financial relationships that could be construed as a potential conflict of interest.

Received: 02 July 2010; paper pending published: 25 July 2010; accepted: 19 November 2010; published online: 06 December 2010.

Citation: Muntean BS, Horvat CM, Behler JH, AbouAlaiwi WA, Nauli AM, Williams FE and Nauli SM (2010) A comparative study of embedded and anesthetized zebrafish in vivo on myocardiac calcium oscillation and heart muscle contraction. Front. Pharmacol. 1:139. doi: 10.3389/ fphar.2010.00139

This article was submitted to Frontiers in Cardiovascular and Smooth Muscle Pharmacology, a specialty of Frontiers in Pharmacology.

Copyright (c) 2010 Muntean, Horvat, Behler, AbouAlaiwi, Nauli, Williams and Nauli. This is an open-access article subject to an exclusive license agreement between the authors and the Frontiers Research Foundation, which permits unrestricted use, distribution, and reproduction in any medium, provided the original authors and source are credited. 